\title{
Estimation of attractor dimension of EEG using singular value decomposition
}

\author{
N PRADHAN ${ }^{1 *}$, D NARAYANA DUTT ${ }^{2}$, P K SADASIVAN $^{1}$ and \\ R G RAMESH ${ }^{1}$
}

${ }^{1}$ Department of Psychopharmacology, National Institute of Mental Health and Neurosciences, Bangalore 560 029, India

${ }^{2}$ Department of Electrical Communication Engineering, Indian Institute of Science, Bangalore 560 012, India

\begin{abstract}
This paper describes a novel application of singular value decomposition (SVD) of subsets of the phase-space trajectory for calculation of the attractor dimension of a small data set. A certain number of local centres $(M)$ are chosen randomly on the attractor and an adequate number of nearest neighbours $(q=50)$ are ordered around each centre. The local intrinsic dimension of a local centre is determined by the number of significant singular values and the attractor dimension $\left(D_{2}\right)$ by the average of the local intrinsic dimensions of the local centres. The SVD method has been evaluated for model data and EEG. The results indicate that the SVD method is a reliable approach for estimation of attractor dimension at moderate signal to noise ratios. The paper emphasises the importance of SVD approach to EEG analysis.
\end{abstract}

Keywords. Nonlinear dynamics; chaos; EEG; SVD; phase-space; attractor dimension.

\section{Introduction}

The field of nonlinear dynamics or chaos has undergone explosive growth in the last few years. Applications have been made in many diverse fields including physics, chemistry, fluid dynamics, meteorology, economics, medicine and sociology (Lin 1984; Thomson \& Stewart 1987). One aspect of nonlinear dynamics involves time series analysis. Electroencephalogram (EEG) is a time series of the electrical activity of the brain. It is regarded as a paraphenomenon of integrated metabolic processes of the brain. It reflects the activities in the underlying brain structures and particularly that of the cerebral cortex below the scalp surface. EEG is one of the commonly used noninvasive tools for studying brain functions. The frequency domain representation of EEG has been in use for over half a century to discern the various patterns of brain activity in relation to behavioural states. In this, a signal block is taken and then Fourier-transformed to get the power spectral estimates

\footnotetext{
*Author for correspondence
} 
which has led to the familiar alpha, beta, delta and theta components. The alpha actvity represents EEG activity in the frequency range 8-13 Hz. It is seen during eye-closed relaxed states. It is predominantly seen in the occipital region of the scalp. EEG activity in the frequency range $13-30 \mathrm{~Hz}$ is termed as beta rhythm and characterises aroused and vigilant state of behaviour. The theta $(4-8 \mathrm{~Hz})$ activities are observed during drowsiness and in pathological conditions. Similarly the delta activities $(0.5-4 \mathrm{~Hz})$ are seen during deep sleep. However, these never reflect the dynamicity of the underlying process of a frequency band into which maximum power is concentrated. On the contrary the newly developed method of phase-space representation can show the dynamics of a system as it evolves with time. With emergence of methods for handling time series generated by nonlinear dynamics, a different set of descriptive measures are now available. The progress in the theory of nonlinear dynamics in the past decade has made it possible to think of modelling the brain as a continuous, spontaneously changing, nonlinear dynamical system (Baser 1983). Grassberger \& Procaccia (1983a, 1983b) have further developed the tools to model experimental data as the output of nonlinear dynamical system. The study involving EEG falls in this category. These new concepts for the investigation of microscopic properties of brain activity offer a fresh way to provide new explanations (Pool 1989). For example let us consider the changing pattern of EEG as a person goes from wakefulness to drowsiness to deep sleep. The brain activity in waking state is desynchronised and with eye closure partial synchrony (alpha rhythm, $8-13 \mathrm{~Hz}$ activity) is observed. It can be seen that as drowsiness starts and sleep gradually ensues, various transitional states appear as stage 1, stage 2, stage 3 and stage 4 in the sleep cycle (Rechtschaffen \& Kales 1968). The brain activity evolves from random-like behaviour to more periodic activity with increasing depth of slow wave sleep. It is broken by intermitteni bursts of desynchronised activity of varying durations called REM (rapid eye movement) during which dreams occur. A similar form of synchronised slow wave activity is seen in anaesthesia. A deep state of anaesthesia is marked by low amplitude and highly periodic slow wave activity. The spike and wave activity during seizure discharge is a high frequency periodic activity of the neurons and the specific waveforms are repeated for a short duration. There are reports of high alpha activity and increased coherence during various meditative states. The psychotic states have not yielded any definite EEG patterns. Even the maturational status of brain may be reflected in the infant EEG. Cognitive activites or intensive mental tasks have not produced any discerning patterns in EEG different from background activity. It is now believed that nonlinear dynamical methods of analysis may discern these states of neural activity. It may be safely assumed that the brain's dynamical behaviour is well reflected in EEG. Therefore, the nonlinear measures have found applications in the analysis and interpretation of EEG and the seemingly random nature of EEG is thought to be due to chaotic neuronal activities. Calculation of attractor dimension or correlation dimension has dominated recent literature and has been used to characterise the effects of anaesthesia, epileptic discharge and mental activity (Babloyantz \& Destexhe 1986; Layne et al 1986; Mayer-Kress \& Layne 1987; Watt \& Hameroff 1987; Nan \& Jinghua 1988).

The dimension of the attractor is a characteristic feature of the underlying neuronal process generating the EEG signals. The dimension value of the attractor is of significance in feature detection of various brain states, classification of patterns of neural activities, differentiation of various types of neural activities and identification of specific drug effects on the brain. The attractor dimension directly reflects the degrees of freedom of the system under study. Therefore, nonlinear dynamics provides a model for signal generation and temporal prediction which may help in determining the nature of neuronal 
processes governing the state of brain activity. Data on attractor dimension are available in literature from subjects in normal resting states. during seizures and in stages of sleep and states of anaesthesia. Correlation dimension analysis is also available from cases with attentional tasks in humans and experimental learning situations in rats. Human data on attractor dimension in resting states have been rather less consistent with a range from 3 to over 10. The dimensionality has been seen to drop during an epileptic epoch consistently. The difficulties in comparing disparate results arise because of the algorithms used and the definition of dimensionality employed. Certain agreed upon uniform conventions may emerge for handling experimental time series in future. However, using the parameter of attractor dimension, a specific predictive model can be built and experimental verification of the model is possible. This newly gained insight on the chaotic dynamics of the brain is a significant departure from the earlier stochastic visualisation. It appears that nonlinear dynamics is going to be the method of study of complex systems and their experimental time series. The application of nonlinear methods to EEG analysis has been discussed in detail in a previous paper (Pradhan \& Narayana Dutt 1993).

The overall interpretation of an EEG record is based on a qualitative impression about the changing patterns in EEG activity. The study utilises the singular value decomposition (SVD) of a subset of phase space trajectory to evaluate the attractor dimension. The SVD method has been applied to model data from Henon and Lorenz maps. As EEG records are invariably noisy, various levels of noise have been added to the model data to evaluate the suitability of the method for its application to experimental time series like EEG. The method has also been applied to real EEG data having alpha, beta, theta, delta and indeterminate activities for different data lengths $(512,1024.2048,4096,8192,16384$ and 20480 points) to evaluate the data requirement of the SVD method for its application to EEG analysis.

\section{Extracting attractor dimension}

The construction of a phase-space trajectory is a crucial step in nonlinear analysis of EEG time series where the unidimensional voltage data are transformed to its trajectory in a multidimensional phase-space. Temporally experimental time series are single-dimensional data. At any given instant of time it has only one phase variable. Before the turn of the century, Poincare showed that much can be learnt about dynamical behaviour from the analysis of trajectories in a multidimensional phase-space in which a single point characterises the entire system at an instant of time. The experimentalist's dilemma has been that for a system with $N$ degrees of freedom it seems necessary to measure $N$ independent variables, an almost impossible chore for complex systems. The problem found a solution in the embedding theorem of Takens (Pradhan \& Narayana Dutt 1993) that a multidimensional phase-space can be constructed from measurement of a single variable (like the electrical potential). For a time series $V\left(t_{i}\right), i=0,1,2,3, \ldots, N$, the phase-space vector $\mathbf{x}(t)$ is constructed by assigning coordinates

$$
\begin{aligned}
x_{1}(t) & =V(t) \\
x_{2}(t) & =V(t+T) \\
\vdots & \\
\vdots & \\
x_{d}(t) & =V(t+(d-1) T),
\end{aligned}
$$


where $T$ is a delay time and $d$ is the embedding dimension. The lag or delay $T$ may be determined by the first zero of the corresponding autocorrelation function (Liebert \& Schuster 1989). On construction of multidimensional phase-space vectors from a time series, the dynamical parameter, attractor dimension $\left(D_{2}\right)$ or correlation dimension may be calculated.

Dimension is perhaps the most basic property of an attractor (Farmer et al 1983). To characterise an attractor, one has to determine its dimension. One classical estimation of attractor dimension is provided by the algorithm of Grassberger \& Procaccia (1983a, 1983b) (GPA) which measures for each embedding dimension $d$, the number of couples of $\left(x_{i}, x_{j}\right)$ whose distance is less than a given radius $r$. More precisely, the algorithm computes the correlation integral $C(r)$ given by

$$
C(r)=\frac{1}{N^{2}} \sum_{i=1}^{N} \sum_{j=1, j \neq i}^{N} \theta\left(r-\left|x_{i}-x_{j}\right|\right),
$$

where $\theta$ is the Heaviside function.

Grassberger \& Procaccia (1983a, 1983b) showed that the correlation integral $C(r)$ obeys the following scaling law:

$$
C(r) \longrightarrow r^{D_{2}}
$$

Therefore,

$$
D_{2}=\lim _{r \rightarrow 0} \frac{\log C(r)}{\log r} .
$$

Here, $C(r)$ is a measure of the probability that two arbitrary points $x_{i}$ and $x_{j}$ of the phase will be separated by distance $r$. The main point is that $C(r)$ behaves as a power of $r$ for small $r$. Therefore plotting $\log C(r)$ versus $\log (r)$ allows us to calculate $D_{2}$ from the slopes of the curves. If the slopes of the graphs for increasing embedding dimensions converge to a saturation value, this limit is called the correlation dimension $D_{2}$. For a stochastic process, there is no convergence and the slope keeps on increasing with increase in embedding dimension.

The correlation dimension is introduced in information theory and is a generalization of the Hausdorff (or fractal) dimension $D_{0}$. Moreover, it estimates the information dimension $D_{1}$ when $D_{0}>D_{1}>D_{2}$. GPA is one of the widely used method for determination of attractor dimension Grassberger \& Procaccia (1983a, 1983b) where highend computing facilities are available.

It can be seen that for data length of $N$, GPA needs a time of order $N^{2}$ and it is further enhanced if estimates are made for an entire range of embedding dimensions. An optimised method of estimating correlation dimension has been reported by Grassberger (1990). The GPA has also been modified by investigators for efficient computation of $D_{2}$ (Dvorak 1990; Theiler 1986). The GPA implemented on a Workstation (HP 9000/735) takes nearly 45 minutes for calculation of the correlation integrals for embedding dimensions 3 to 12 for only 1024 data points. It takes several hours for 40000 data points on the same machine. Moreover, GPA requires large data points, 80000 or more, for a reliable estimation of correlation dimension. GPA fares poorly in presence of noise where the plot of $\log C(r)$ vs $\log (r)$ may not converge. Further, the scaling region for determining the slope (hence $D_{2}$ ) is more often arbitrarily fixed and thus giving rise to varying results by different investigators. Therefore, we have investigated the SVD method which is computationally efficient and there is no arbitrariness of defining the scaling region. 


\section{Singular value approach for estimation of attractor dimension}

The SVD method has been used for dimension estimation of chaotic attractors (Broomhead \& King 1986; Passamante et al 1989). In this study, we describe a novel application of SVD for analysis of EEG signals. This approach is computationally efficient in comparison to standard GPA considering the massiveness of EEG data. The procedure of singular value approach is an extension of the idea of local intrinsic dimensionality introduced by Fulunga \& Oslen (1971). The underlying idea is to make use of local linear approximation to the nonlinear evolution governing the dynamics. Given an embedding dimension $d$, a number of local centres $M$ is selected randomly on the attractor. For each of these local centres $\mathbf{x}_{i}$, the $q$ nearest neighbours are retained and organised in a $d \times q$ matrix as

$$
\mathbf{X}=\left(\mathbf{x}_{1}, \mathbf{x}_{2}, \mathbf{x}_{3}, \ldots, \mathbf{x}_{q}\right) \text {. }
$$

The rank of $\mathbf{X}$ is determined using SVD. The significant singular values, or equivalently, the eigenvalues, are known to produce the dimensionality of the corresponding data space. The number of significant singular values then represents the local intrinsic dimension of the attractor at its local centre in phase-space. The singular value spectrum contains both the signal subspace and the noise subspace information. In the absence of noise, the limiting smallest value of singular values depends upon the number of nearest neighbours taken for construction of the matrix $\mathbf{X}$ around the local centre. Therefore, a certain minimum number of nearest neighbours are essential for obtaining significant singular values for estimation of the local intrinsic dimension of a local centre. Similarly adequate number of local centers are essential to cover the entire phase-space map for a reliable estimation of the attractor dimension (Pike 1987).

The question of deciding which singular values are significant can also be a problem. The point where one places the threshold between signal and noise must be carefully chosen. The selection of the threshold is a matter of judgement which in some cases may be difficult. More objective methods for setting the threshold are being investigated. The difficult problem is the separation of the noise singular values from the signal singular values, particularly for low signal to noise ratios (SNR). The selection of the threshold will, in general, depend on the distribution or statistics of the noise singular values. However, at moderate SNR the number of significant singular values represent the dimension of signal subspace. The significant singular values may be obtained by using a threshold criterion of $30 \%$ of the maximum of the singular value spectrum.

Once singular values have been estimated for various local centres on the attractor, the average of all local intrinsic dimensions is calculated as the attractor dimension.

$$
D_{2}=P_{1} s_{1}+P_{2} s_{2}+\ldots+P_{r} s_{r}
$$

where $s_{1}=1, s_{2}=2, \ldots, s_{r}=r$ and $r$ is the maximum number of significant singular values for $M$ local centres. $P_{i}$ is the estimated probability of $s_{i}$ occurring i.e., $P_{i}=h_{i} / M$ with $h_{i}$ being the number of local regions that have dimension $s_{i}$, and $M$ being the total number of local regions. There are certain advantages of the SVD approach. It is computationally less intensive and can be applied to both small and large data sets. However, the results are more accurate with 20000 or more data points. It is feasibly implemented on a Workstation and does not require expensive high computing platforms. A consistent criteria of determining the threshold for significant singular values may be applied instead of an arbitrary scaling region as in the case of GPA. In the same system it takes only 40 seconds to calculate the attractor dimension of 40000 points with an 
embedding dimension of 30 , local centres on the attractor $M=50$ and number of nearest neighbouring vectors at the local centre $q=50$. The method gives a reliable estimate of attractor dimension for noisy data. It has the potential of being implemented on-line in EEG monitoring laboratories.

\section{Application of SVD approach to model data}

The well known Henon map and Lorenz map are classical examples of chaotic systems. The SVD approach is presented for the Lorenz and Henon attractors. The Lorenz system is given by

$$
\begin{aligned}
& \dot{x}=c y-c x, \\
& \dot{y}=r x-y-x z, \\
& \dot{z}=b z+x y,
\end{aligned}
$$

where $c=10, b=8 / 3, r=28$. Gills routine was used for integration with a step size of 0.006 . The initial conditions $x(0)=y(0)=z(0)=1$ were used for the Lorenz map. 200000 data points were generated of which initial 2000 points were discarded to remove the initial transients.

The Henon map is given by

$$
x_{i+1}=1-a x_{i}^{2}+b x_{i-1},
$$

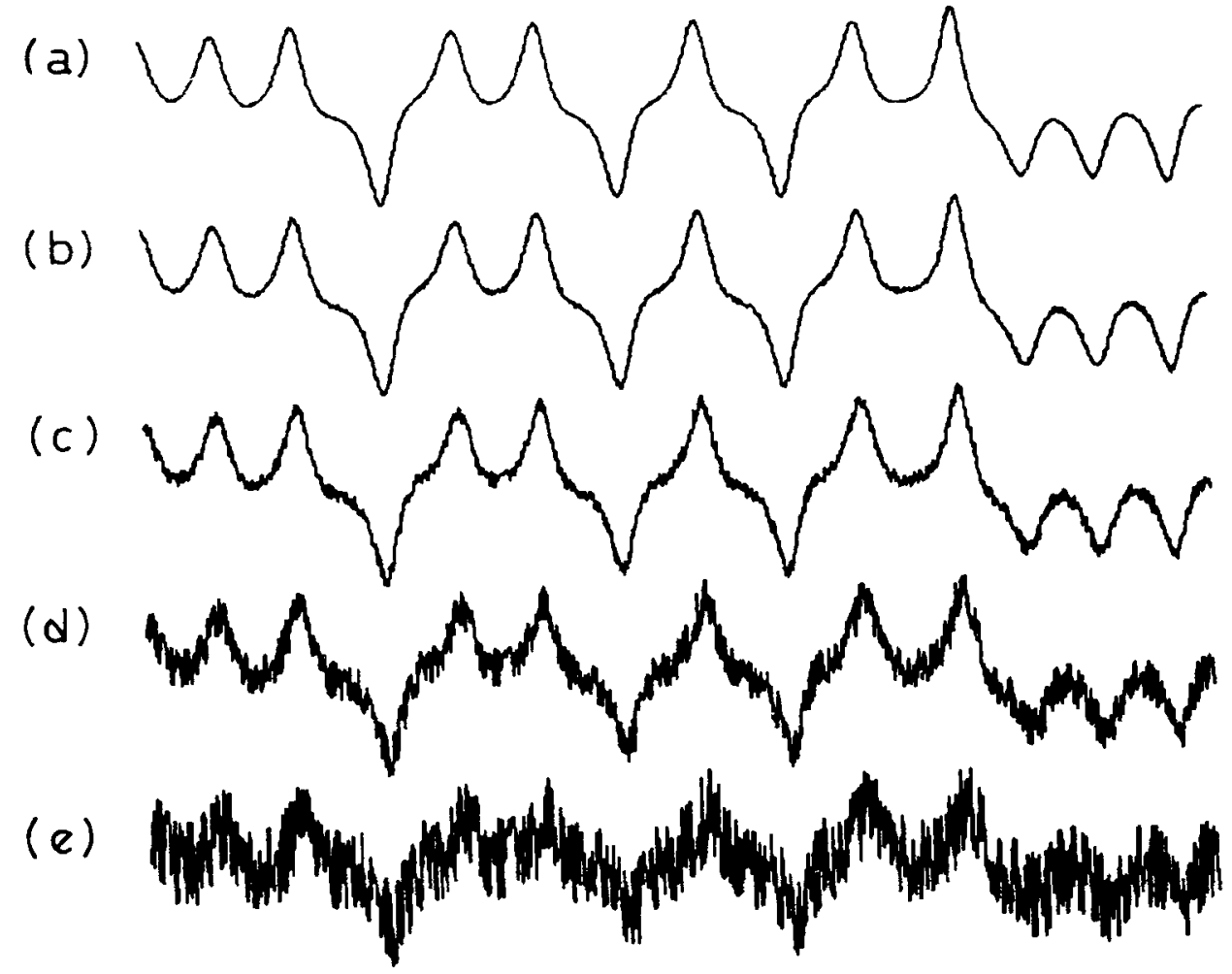

Figure 1. Time plot of Lorenz map. White Gaussian noise has been added to 2048 data points (a) infinite, (b) $30 \mathrm{~dB}$, (c) $20 \mathrm{~dB}$, (d) $10 \mathrm{~dB}$ and (e) $0 \mathrm{~dB}$ SNR. 
(a)

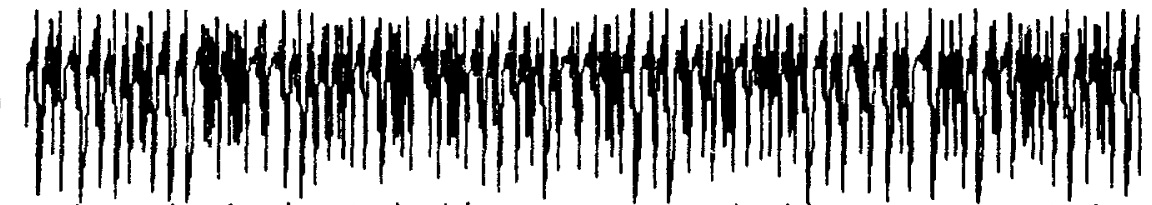

(b)

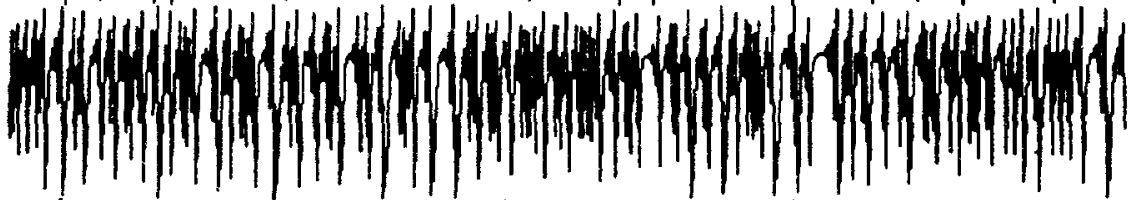

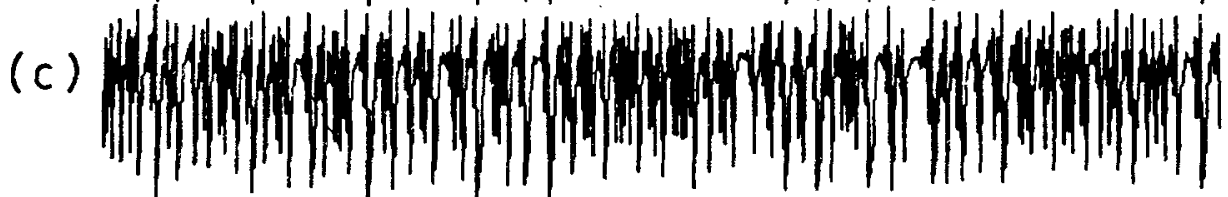

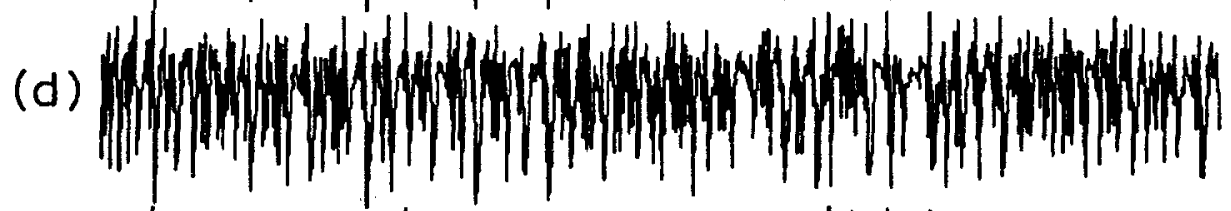

(e)

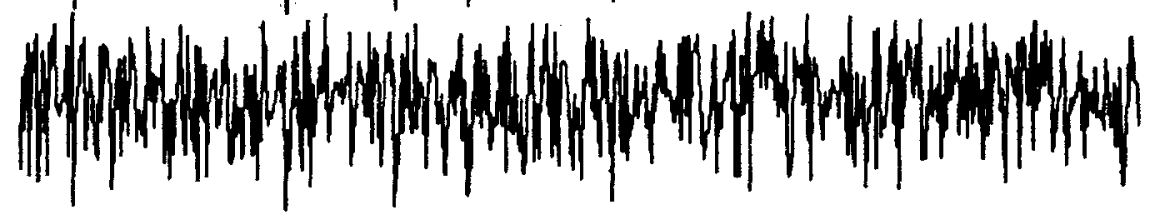

Figure 2. Phase-space plots of Lorenz map with additive noise (a) infinite, (b) $30 \mathrm{~dB}$, (c) $20 \mathrm{~dB}$, (d) $10 \mathrm{~dB}$ and (e) $0 \mathrm{~dB}$ SNR.

where $a=1.4$ and $b=0.3$. Data were generated using the initial conditions $x_{i}=x_{i-1}=0$ for the Henon map data consisting of 900000 points. The initial 2000 data points were discarded to avoid transients at the beginning of the data.

We have used varying lengths of data segments $(512,1024,2048,4096,8192,16384$ and 20480). With an embedding dimension of 10 , phase space vectors were created. The number of local centres $M=50$ were randomly chosen on the attractor. We obtained 50 nearest neighbouring points $(q=50)$ around each local centre. The matrix $\mathbf{X}$ was generated using the nearest neighbour points for a given local centre using (5). The SVD of $\mathbf{X}$ gave the singular values. Using the threshold criteria of $30 \%$ of the maximum singular value, the number of significant singular values were determined for a given local centre. The attractor dimension was determined as per (6). The estimations have been carried out with various levels of additive noises (infinite, $0 \mathrm{~dB}, 10 \mathrm{~dB}, 20 \mathrm{~dB}$ and $30 \mathrm{~dB}$ ) to the Henon and Lorenz maps. A Gaussian random sequence of zero mean and unit variance was generated and added to the model data for various levels of SNR.

$$
\mathrm{SNR}(\mathrm{dB})=10 \log \left[\frac{\text { signal power }}{\text { noise power }}\right] \text {. }
$$

The sample data segments and their phase space plots for Henon and Lorenz maps with additive noise are given in figures $1-4$. The saturation of dimension estimate with increasing number of local centres for the Lorenz map has been given in figure 5. It shows that $D_{2}$ value may be reliably estimated by fixing $M$ and $q$ at 50 .

The results of SVD method for model data have been summarised in tables 1 and 2 which depict the relationships of data lengths, noise levels and number of local centres with 
(a)

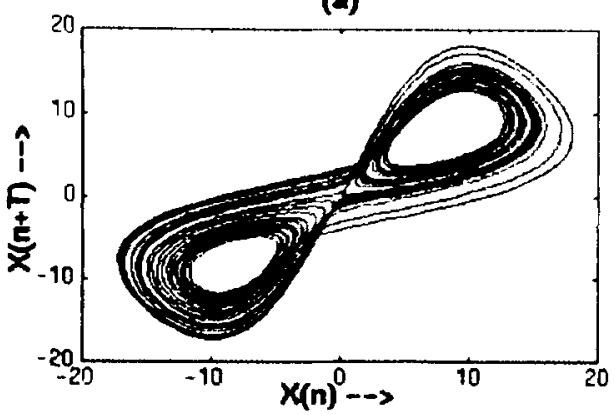

(c)

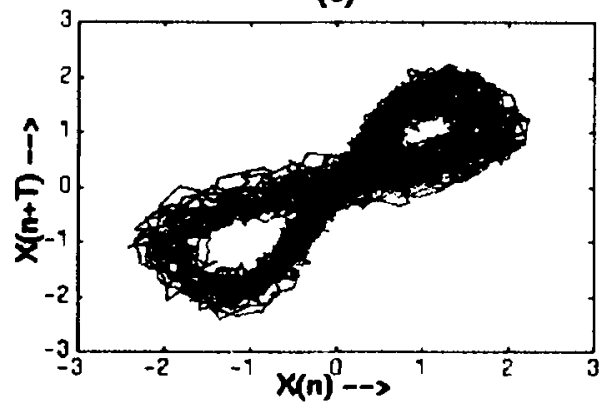

(a)

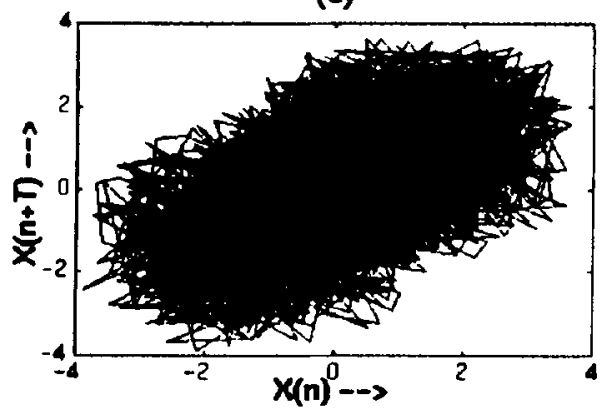

(b)

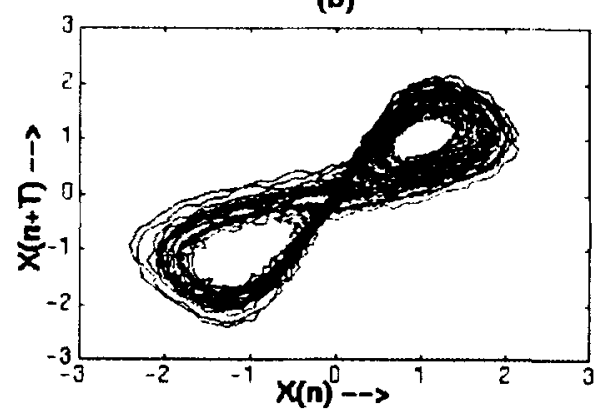

(d)

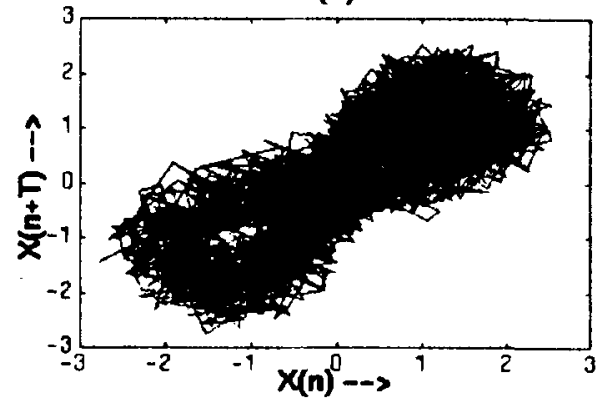

Figure 3. Time plot of Henon map. White Gaussian noise has been added to 2048 data points (a) infinite (b) $30 \mathrm{~dB}$ (c) $20 \mathrm{~dB}$ (d) $10 \mathrm{~dB}$ and (e) $0 \mathrm{~dB}$.

attractor dimensions. The values are the averages of dimensions calculated on consecutive data segments.

\section{Application of SVD approach to EEG data}

Oscillations in single neuron and neuronal ensembles underlie the generation of various pattern features in EEG. Since mathematicians have long known that the periodic forcing of nonlinear oscillators can give rise to complex phase-locking patterns, bifurcations and aperiodic dynamics (Hayashi et al 1982), one anticipates that such behaviour might be observable in forced neuronal oscillators. Studies on the periodic forcing of biological oscillators have, in fact, been interpreted in the context of chaotic dynamics (Hayashi et al 1982). It has been proposed that complex EEG patterns which occur normally arise from interactions between a large number of neural relaxation oscillators (Baser 1980). All these observations raise the possibility that some of the observed variability in neural electrical activity may be a reflection of intrinsically chaotic dynamics. Thus the concept of chaos introduces a perspective for the analysis of neural dynamics and this has been the motivation for the present study. EEG, being a complex pattern generated in the brain by a 
(a)

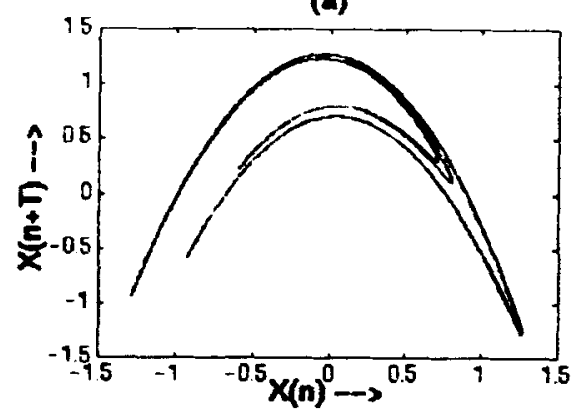

(c)

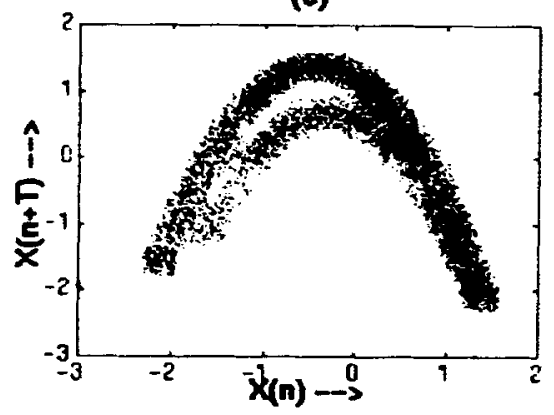

(c)

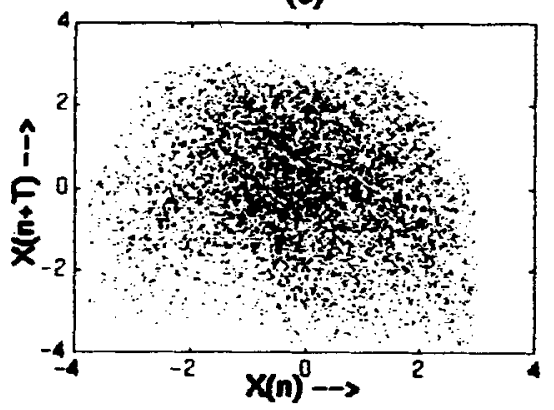

(b)

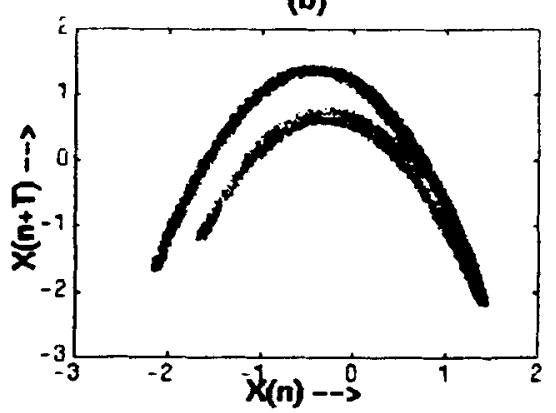

(d)

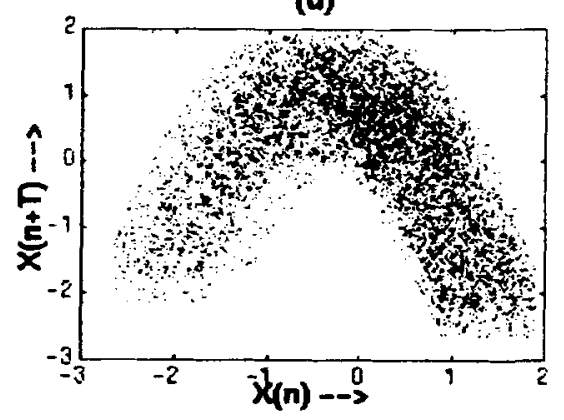

Figure 4. Phase-space plots of Henon map with additive noise (a) infinite, (b) $30 \mathrm{~dB}$, (c) $20 \mathrm{~dB}$, (d) $10 \mathrm{~dB}$ and (e) OdB SNR.

possible chaotic process seemingly very irregular and random-like in character, is in fact a good choice for application of chaotic dynamics. Estimation of attractor dimension is a fundamental measurement for characterising chaotic systems. Keeping this in view, we have applied the SVD method for estimating the attractor dimensions of changing patterns in EEG.

\subsection{Recording and digitisation of EEG}

The EEG signals from 8 volunteers were recorded with Nihon Kohden EEG amplifiers. The four channels of unipolar EEG, $F_{3}$ (chl-1), $F_{4}(\mathrm{chl}-2), 0_{1}(\mathrm{chl}-3), 0_{2}(\mathrm{chl}-4)$ referenced to $A_{2}$ were obtained for durations varying from 10 to 15 minutes. The subjects were instructed to close their eyes for some time during the data acquisition period and allowed to sleep. The signals were digitised at $128 \mathrm{samples} / \mathrm{second} / \mathrm{channel}$. Data acquisition was accomplished with the use of 12-bit DT-2841 ADC coupled with DT-7020 array processor (Data Translation Inc, MA, USA) in a PC-AT computer. Data were then transferred over a network (off-line) to HP9000/735 Graphics Workstation for further analysis. The raw EEG signals were filtered through a bandpass $(0.25-32 \mathrm{~Hz})$ 4th order Butterworth filter twice cascaded. The data were scanned for a specific activity and 25600 consecutive points 


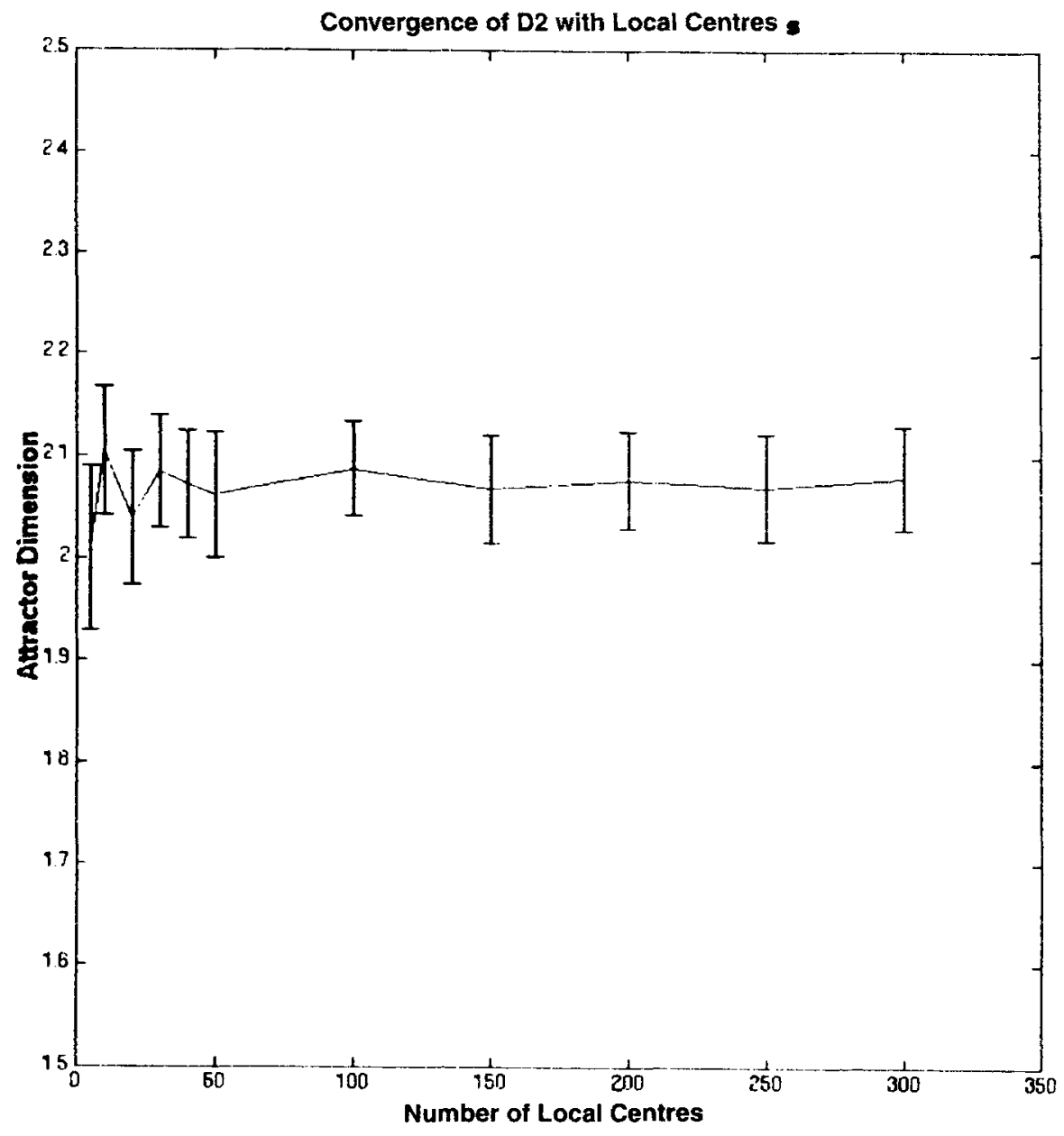

Figure 5. Saturation curve of D2 with increasing number of local centres $(M)$ for Lorenz map $(N=4096, q=50)$.

having a given pattern of EEG activity were extracted. The extracted EEG segments were then processed for quantification of attractor dimension. The time series plots of various EEG activities are shown in figure 6.

\subsection{Estimation of attractor dimension of EEG data}

The dynamical behaviour of the brain is currently being viewed in the perspective of nonlinear dynamics. There are several reports of low dimensional chaotic activity in various states of human behaviour (Baser 1980). While dimension estimate provides a measure for classifying various brain activities, the Lyapunov exponent may be seen as a powerful estimate of dynamics of the system reflecting the long-time average exponential rates of divergence or convergence of nearby trajectories in state space. If a system has at least one positive Lyapunov exponent, then the system is chaotic. It stems from the premise (conjecture) that chaotic systems are highly sensitive to initial conditions. It implies that small changes in the state of a chaotic system grow exponentially and dominate the system behaviour (Wolf et al 1985). Since the error bar is composed of many adjacent states in the solution space or phase space and adjacent states diverge quickly, it follows that error bars 
(a)

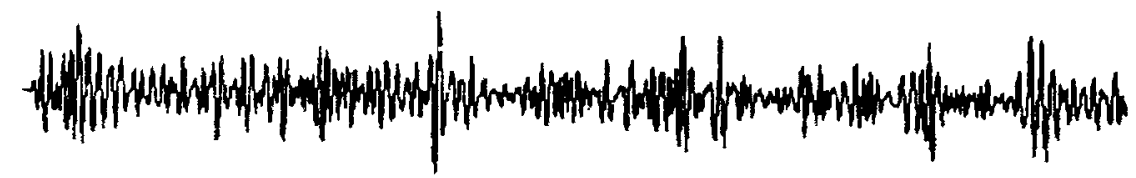

(b)

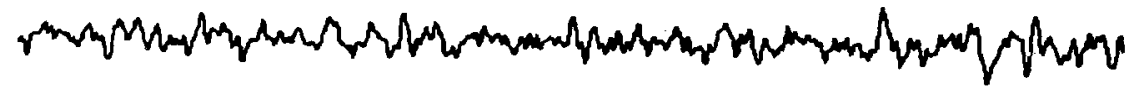

(c)

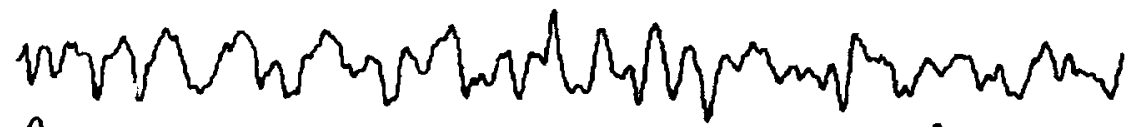

(d)
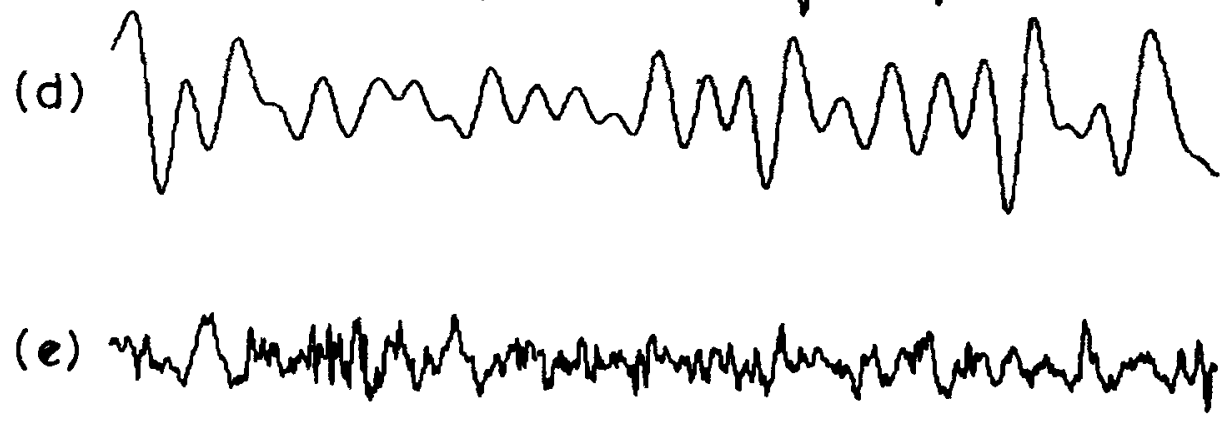

Figure 6. Time plot of (a) alpha, (b) beta, (c) theta, (d) delta and (e) indeterminate EEG activity of 8 s duration.

on the initial conditions of chaotic systems grow exponentially fast. Error bars on initial conditions are omnipresent, so it may be concluded that long-term prediction of chaotic systems are futile no matter how the system prediction is implemented. The dominant Lyapunov exponent $\left(\lambda_{1}\right)$ has also been evaluated by application of the algorithm of Wolf et al (1985). The $\lambda_{1}$ values of different EEG activities are seen to be positive. The value of $\lambda_{1}$ is $0.143 \pm 0.01$ for alpha activity. For beta activity the $\lambda_{1}$ value is $1.801 \pm 0.10$ and for theta, delta and indeterminate activities the values are $0.162 \pm 0.13,0.135 \pm 0.01$, and $1.102 \pm 0.01$ respectively. It implies that EEG is chaotic.

The attractor dimension for varying lengths of data $(512,1024,2048,4096,8192,16384$ and 20480 points) obtained for alpha, beta, theta, delta and indeterminate activities is given in tabie 3. The phase-space plots of various EEG activities have been presented in figure 7. The number of nearest neighbours $q=50$ has been used in computing the dimension for local centres $M=50, M=100$ and $M=200$ as in the earlier case. The maximum embedding dimension is 30 . From the results of Lorenz and Henon maps, it is evident that 4096 to 8192 data points are the optimum data length covered by $M=50$ local centres. Here we have used 4096 data points as optimum length for estimation of attractor dimension.

\section{Results and discussion}

The analysis of EEG in the past several decades has been attempted by the phenomenological approach, in which the EEG is seen to be a band-limited signal produced by some black-box with unknown or white Gaussian noise input. More recently a model-based 
(a)

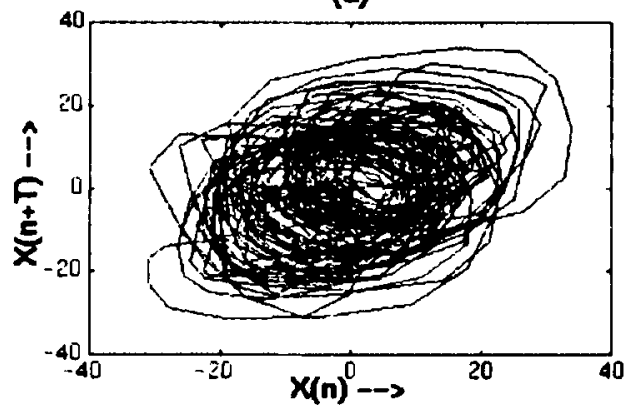

(c)

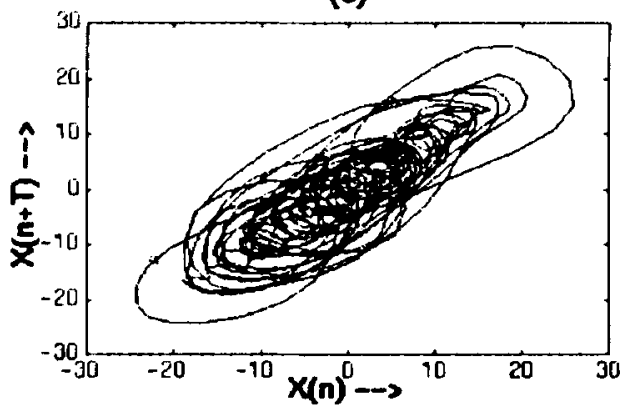

(e)

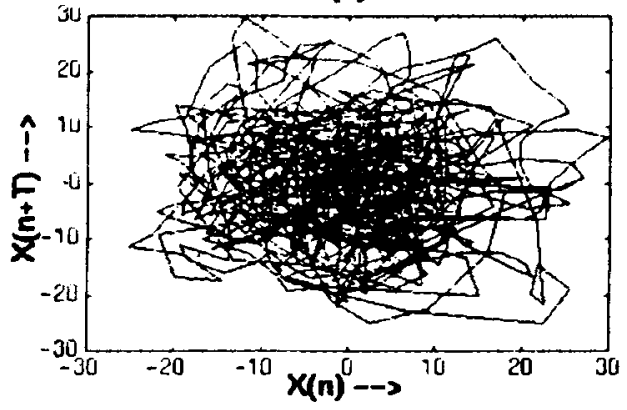

(b)

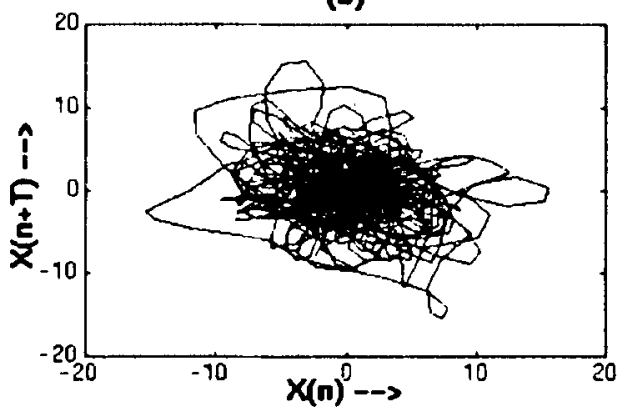

(d)

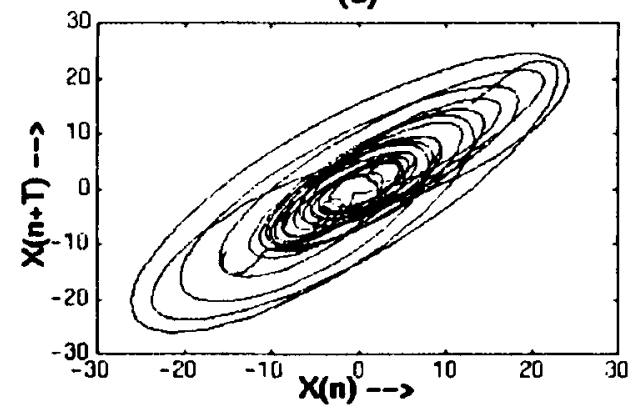

Figure 7. Phase-space plots of (a) alpha, (b) beta, (c) theta, (d) delta and (e) indeterminate EEG activities.

approach, incorporating concepts developed in the area of nonlinear dynamics and chaos theory, has been used. The application of nonlinear dynamics to EEG analysis may provide information to understand the underlying neurodynamics of EEG generation and its evolution. Estimation of the attractor dimension is a primary step in this direction. The GPA has been in the forefront of the computational procedures for obtaining the dimension of the attractor. For reasons already mentioned, an alternate method suitable for EEG data is called for. We have described a method of application of singular value spectrum for estimating the dimension of the attractor. It was essential to apply the method for model data for determining data lengths suitable for analysis.

We have presented the SVD method for estimation of the attractor dimension of model data from which we extract the information about the appropriate data length requirement for a given number of local centres $(M)$ and number of nearest neighbours $(q)$. The three parameters $M, q$ and $N$ influence the dimension estimate. Therefore, for an optimal evaluation of the dimension, two of the parameters may have to be fixed while the third one is varied. It could be seen that the method is suitable for small data and a suitable estimate of dimension may be obtained with $N=4096-8192$ while $M$ and $q$ are fixed at 50 .

Without additive noise, 512 data points of the Lorenz map yields an attractor dimension of 2.443 which is higher than the theoretically expected dimension (2.01). The expected 
Table 1. Attractor dimension values for Lorenz map.

\begin{tabular}{|c|c|c|c|c|}
\hline \multirow[t]{2}{*}{ SNR } & \multirow[t]{2}{*}{ Data length } & \multicolumn{3}{|c|}{ Attractor dimension } \\
\hline & & $(M=50)$ & $(M=100)$ & $(M=200)$ \\
\hline \multirow[t]{7}{*}{$\infty$} & 512 & 2.443 & 2.417 & 2.498 \\
\hline & 1024 & 2.510 & 2.558 & 2.579 \\
\hline & 2048 & 2.257 & 2.174 & 2.156 \\
\hline & 4096 & 2.033 & 1.860 & 1.879 \\
\hline & 8192 & 1.783 & 1.810 & 1.873 \\
\hline & 16384 & 1.520 & 1.470 & 1.580 \\
\hline & 20480 & 1.435 & 1.440 & 1.435 \\
\hline \multirow[t]{7}{*}{$30 \mathrm{~dB}$} & 512 & 2.442 & 2.506 & 2.494 \\
\hline & 1024 & 2.514 & 2.560 & 2.602 \\
\hline & 2048 & 2.272 & 2.126 & 2.141 \\
\hline & 4096 & 2.029 & 1.900 & 1.896 \\
\hline & 8192 & 1.774 & 1.830 & 1.870 \\
\hline & 16384 & 1.518 & 1.520 & 1.525 \\
\hline & 20480 & 1.433 & 1.460 & 1.455 \\
\hline \multirow[t]{7}{*}{$20 \mathrm{~dB}$} & 512 & 2.519 & 2.576 & 2.528 \\
\hline & 1024 & 2.518 & 2.625 & 2.597 \\
\hline & 2048 & 2.282 & 2.164 & 2.138 \\
\hline & 4096 & 2.019 & 1.932 & 1.877 \\
\hline & 8192 & 1.767 & 1.705 & 1.790 \\
\hline & 16384 & 1.501 & 1.560 & 1.510 \\
\hline & 20480 & 1.411 & 1.438 & 1.420 \\
\hline \multirow[t]{7}{*}{$10 \mathrm{~dB}$} & 512 & 2.875 & 2.744 & 2.777 \\
\hline & 1024 & 2.772 & 2.881 & 2.918 \\
\hline & 2048 & 2.510 & 2.294 & 2.299 \\
\hline & 4096 & 2.179 & 2.066 & 2.064 \\
\hline & 8192 & 1.842 & 1.965 & 1.858 \\
\hline & 16384 & 1.486 & 1.580 & 1.558 \\
\hline & 20480 & 1.374 & 1.430 & 1.439 \\
\hline \multirow[t]{7}{*}{$0 \mathrm{~dB}$} & 512 & 8.260 & 8.136 & 8.133 \\
\hline & 1024 & 7.666 & 7.979 & 7.969 \\
\hline & 2048 & 6.883 & 6.629 & 6.676 \\
\hline & 4096 & 5.423 & 5.424 & 5.328 \\
\hline & 8192 & 3.842 & 4.165 & 3.948 \\
\hline & 16384 & 2.449 & 2.360 & 2.435 \\
\hline & 20480 & 2.059 & 1.992 & 2.025 \\
\hline
\end{tabular}

value could be reached when the data points are about 4096 . With increase in the number of points beyond 4096, the number of local centres $(M=50)$ are not adequate for covering the entire phase-space; therefore there is a drop in the value of the dimension. When 50 
Table 2. Attractor dimension values for Henon map.

\begin{tabular}{|c|c|c|c|c|}
\hline \multirow[t]{2}{*}{ SNR } & \multirow[t]{2}{*}{ Data length } & \multicolumn{3}{|c|}{ Attractor dimension } \\
\hline & & $(M=50)$ & $(M=100)$ & $(M=200)$ \\
\hline \multirow[t]{7}{*}{$\infty$} & 512 & 6.214 & 6.222 & 6.232 \\
\hline & 1024 & 4.318 & 4.314 & 4.336 \\
\hline & 2048 & 2.805 & 2.818 & 2.839 \\
\hline & 4096 & 1.787 & 1.768 & 1.776 \\
\hline & 8192 & 1.254 & 1.242 & 1.268 \\
\hline & 16384 & 1.077 & 1.075 & 1.093 \\
\hline & 20480 & 1.056 & 1.050 & 1.040 \\
\hline \multirow[t]{7}{*}{$30 \mathrm{~dB}$} & 512 & 6.220 & 6.210 & 6.210 \\
\hline & 1024 & 4.319 & 4.312 & 4.274 \\
\hline & 2048 & 2.809 & 2.798 & 2.785 \\
\hline & 4096 & 1.785 & 1.750 & 1.803 \\
\hline & 8192 & 1.254 & 1.265 & 1.248 \\
\hline & 16384 & 1.078 & 1.070 & 1.105 \\
\hline & 20480 & 1.050 & 1.020 & 1.032 \\
\hline \multirow[t]{7}{*}{$20 \mathrm{~dB}$} & 512 & 6.291 & 6.279 & 6.260 \\
\hline & 1024 & 4.373 & 4.373 & 4.359 \\
\hline & 2048 & 2.836 & 2.788 & 2.857 \\
\hline & 4096 & 1.795 & 1.760 & 1.796 \\
\hline & 8192 & 1.255 & 1.270 & 1.243 \\
\hline & 16384 & 1.075 & 1.090 & 1.080 \\
\hline & 20480 & 1.048 & 1.060 & 1.060 \\
\hline \multirow[t]{7}{*}{$10 \mathrm{~dB}$} & 512 & 6.880 & 6.873 & 6.864 \\
\hline & 1024 & 4.865 & 4.852 & 4.842 \\
\hline & 2048 & 3.198 & 3.197 & 3.193 \\
\hline & 4096 & 1.993 & 2.026 & 1.996 \\
\hline & 8192 & 1.328 & 1.290 & 1.347 \\
\hline & 16384 & 1.094 & 1.080 & 1.100 \\
\hline & 20480 & 1.061 & 1.060 & 1.062 \\
\hline \multirow[t]{7}{*}{$0 \mathrm{~dB}$} & 512 & 9.321 & 9.340 & 9.348 \\
\hline & 1024 & 8.000 & 8.024 & 8.024 \\
\hline & 2048 & 6.409 & 6.436 & 6.479 \\
\hline & 4096 & 4.790 & 4.764 & 4.838 \\
\hline & 8192 & 3.274 & 3.335 & 3.285 \\
\hline & 16384 & 2.075 & 1.970 & 1.940 \\
\hline & 20480 & 1.779 & 1.720 & 1.715 \\
\hline
\end{tabular}

local centres are used on small data sets, there may be overlap among the attractor zones thereby producing a higher estimate of the dimension. With addition of $0 \mathrm{~dB}$ noise the initial estimate of dimension is 8.2604 for 512 points and it is 5.423 for 4096 points. The phase- 
Table 3. Attractor dimension values for different EEG activities.

\begin{tabular}{|c|c|c|c|c|}
\hline \multirow[t]{2}{*}{ EEG } & \multirow[t]{2}{*}{ Data length } & \multicolumn{3}{|c|}{ Attractor dimension } \\
\hline & & $(M=50)$ & $(M=100)$ & $(M=200)$ \\
\hline \multirow[t]{7}{*}{ Delta } & 512 & 4.642 & 4.641 & 4.637 \\
\hline & 1024 & 4.605 & 4.610 & 4.623 \\
\hline & 2048 & 4.606 & 4.555 & 4.575 \\
\hline & 4096 & 4.416 & 4.430 & 4.420 \\
\hline & 8192 & 4.490 & 4.210 & 4.275 \\
\hline & 16384 & 3.973 & 3.993 & 3.998 \\
\hline & 20480 & 3.897 & 3.923 & 3.922 \\
\hline \multirow[t]{7}{*}{ Theta } & 512 & 7.568 & 7.585 & 7.620 \\
\hline & 1024 & 7.387 & 7.332 & 7.322 \\
\hline & 2048 & 7.062 & 7.201 & 7.158 \\
\hline & 4096 & 6.620 & 6.685 & 6.770 \\
\hline & 8192 & 6.216 & 6.326 & 6.376 \\
\hline & 16384 & 5.770 & 5.880 & 5.985 \\
\hline & 20480 & 5.710 & 5.785 & 5.800 \\
\hline \multirow[t]{7}{*}{ Alpha } & 512 & 10.570 & 10.587 & 10.747 \\
\hline & 1024 & 10.066 & 10.184 & 10.050 \\
\hline & 2048 & 8.382 & 8.589 & 8.664 \\
\hline & 4096 & 7.320 & 7.398 & 7.370 \\
\hline & 8192 & 6.391 & 6.401 & 6.411 \\
\hline & 16384 & 5.880 & 5.923 & 5.921 \\
\hline & 20480 & 4.921 & 5.103 & 5.213 \\
\hline \multirow[t]{7}{*}{ Beta } & 512 & 11.491 & 11.679 & 11.689 \\
\hline & 1024 & 11.030 & 11.030 & 11.050 \\
\hline & 2048 & 10.060 & 9.997 & 10.010 \\
\hline & 4096 & 9.340 & 9.270 & 9.340 \\
\hline & 8192 & 8.570 & 8.890 & 8.610 \\
\hline & 16384 & 8.300 & 7.900 & 7.860 \\
\hline & 20480 & 7.600 & 7.680 & 7.820 \\
\hline \multirow[t]{7}{*}{ Indeterminate } & 512 & 8.616 & 8.664 & 8.638 \\
\hline & 1024 & 8.970 & 8.960 & 8.990 \\
\hline & 2048 & 8.540 & 8.570 & 8.582 \\
\hline & 4096 & 8.050 & 7.970 & 7.920 \\
\hline & 8192 & 7.290 & 7.290 & 7.387 \\
\hline & 16384 & 6.920 & 6.920 & 6.970 \\
\hline & 20480 & 6.820 & 6.440 & 6.660 \\
\hline
\end{tabular}

space plots (figures 2 and 4) maintain the characteristic forms of maps upto 10dB SNR. The saddle-shaped appearance of Henon map is apparent at 10dB SNR. At OdB SNR the structures of the maps are lost. Therefore a spurious estimate of dimension is encountered 
at $0 \mathrm{~dB}$ noise as the noise invades the entire phase-space. Such fallacious estimates can be seen in table 1 where the dimension value is 2.059 at 20480 data points; this almost approximates the expected value. It is implied that as the number of data points increase, the number of local centres need to be increased. The estimations have been repeated for 100 and 200 local centres on the attractor. It is apparent that a choice of 50 local centres suffice only for 4096 points. Our experiments indicate that SVD is able to approach the expected value with $30 \mathrm{~dB}$ noise. Even at $10 \mathrm{~dB}$ noise level, the dimension values for 4096 points is 2.17 which is not much deviated from the expected value. Similar results are seen for the Henon map in table 2. Here data lengths of 8192 points yield values that are close to the expected values. The use of SVD in differentiating the signal space and noise space is known in signal processing. At moderate SNR (upto 10dB) the SVD approach differentiates the signal subspace and the noise sub-space by significant differences in their eigenvalues. Therefore the SVD approach may be suitable for signals like EEG. The information is utilised to evaluate the dimension of EEG data.

Different patterns of EEG activities have been analysed with the application of SVD method. We have presented the analysis results for alpha, beta, theta, delta and indeterminate activities. Varying lengths of data segments $(512,1024,2048,4096,8192,16384$ and 20480) have been used to see whether our primary assumption of determining the record length from the model data is valid. It is seen that EEG results fall into a similar pattern of chaotic signals of Lorenz and Henon maps. It can be seen that the attractor dimension values are high for small data sets (512) and decrease with increase in the length of data similar to Lorenz and Henon maps. For delta, the values of the dimension range from 4.641 to 3.897 for 512 to 20480 data points with 50 to 200 local centres. Using the data length criteria from Lorenz and Henon maps, the dimension value may be fixed at 4.416 for 4096 points and 50 local centres. The attractor dimension thus determined by the SVD approach for delta activity is 4.416 which is similar to the range reported in the literature. Alpha activity has a value of 7.32 for 4096 data points. The attractor dimensions for beta, theta and indeterminate EEG activities have values of 9.93, 6.62 and 8.05 respectively. Different patterns may be discerned by their attractor dimension values. It may be seen (figure 6) that beta and indeterminate activities are more random-like whereas the delta, alpha and theta patterns of EEG tend to become periodic. This is more apparent from the phase-space plots (figure 7). Limit-cycle-like behaviour of delta and theta may be seen in the plot. The degree of complexity of the signal may be qualitatively inferred from its phase-space trajectory. There occurs a loss of complexity of EEG as one moves from a relaxed state of alpha to a state of deep sleep predominated by delta. Such loss of complexity of EEG may also be encountered in seizure discharges and degenerative conditions of the brain. Neurobiological significance of the low and high values of attractor dimension which reflects the degree of complexity needs to be determined by more empirical observations in different brain conditions. This paper only reflects the need for a suitable method that could be computationally efficient for any real-time or on-line application in neurobiological investigations of the brain. We have only presented the data of the known patterns of EEG activity to show that the SVD method can be reliably applied to EEG. These estimates are within the acceptable ranges of the dimension values in keeping with the degree of complexity of the signals. The SVD method for obtaining attractor dimensions to different EEG activities presented in this study may have potential applications in the feature detection of EEG patterns. It may also help in understanding the underlying dynamics of neuronal processes in the generation of EEG. 


\section{Conclusions}

The present study suggests that attractor dimension may be an effective method of determining the degree of complexity of the data and may offer a way for feature detection in EEG. SVD method is effective with small data sets at moderate SNR. Hence, it may be considered as a preferred method of determining the attractor dimension of EEG time series. The complex, disorganised and random-like patterns in EEG such as beta and indeterminate activities have higher dimension values and thus they may reflect a greater degree of complexity in their processes. However, the neurobiological significance of high-dimensional and low-dimensional values in different brain states are to be determined. Further studies are needed to establish the correlations of attractor dimension values to those of physiological functions. The nature of attractors of neuronal systems may be of great help in future for understanding the dynamical properties of brain. In this study, we have seen that complex patterns and more random-like EEG activities have higher attractor dimensions. The alpha, delta and theta patterns that are quasi-periodic have lower dimension values than beta and indeterminate activities. The crucial role of length of data with respect to number of local centres in the calculation of attractor dimension has been emphasised.

The chaotic model of EEG generation i.e. the notion that simple, nonlinear systems can produce complex, almost random looking outputs is very appealing. The fact that EEG appears unpredictable, yet is bounded to a limited frequency and amplitude range with a few basic rhythms and waveforms points to the chaotic aspect of the brain's electrical activity. A study of the analysis of EEG has been made here keeping in mind the nonlinear deterministic nature of EEG.

\section{References}

Babloyantz A, Destexhe A 1986 Low dimensional chaos in an instance of epilepsy. Proc. Natl. Acad. Sci. USA. 83: 3513-3517

Baser E 1980 EEG brain dynamics (Amsterdam: Elsevier-North Holland Biomedical press)

Baser E 1983 Synergetics of neuronal populations: A survey on experiments. In Synergetics of the brain, Springer Series in Synergetics of the Brain, (eds) E Baser, H Flohr, H Haken, A J Mandell (Berlin: Springer Verlag), pp.190-200

Broomhead D S, King G P 1986 Extracting qualitative dynamics from experimental data. Physica D20: $217-226$

Dvorak I 1990 Takens versus multichannel reconstruction in EEG correlation exponent estimates. Phys. Lett. A151: 225-233

Farmer J C, Ott E, York J A 1983 Dimension of chaotic attractors. Physica D7: 153-180

Fukunga K, Oslen D R $1971 \mathrm{An}$ algorithm for finding the intrinsic dimensionality of data. IEEE Trans. Comput. C-20: 176-183

Grassberger P 1990 An optimised box-assisted algorithm for fractal dimensions. Phys. Lett. A148: $63-68$

Grassberger P, Procaccia I 1983a Characterisation of strange attractors. Phys. Rev. Lett. 50: 346349

Grassberger P, Procaccia I 1983b Measuring the strangeness of strange attractors. Physica D9: 189-208

Hayashi C 1964 Nonlinear oscillations in physical systems (New York: McGraw Hill)

Hayashi N, Nakao M, Hirakawa N 1982 Chaos in self-sustained oscillation of an excitable biological membrane under sinusoidal stimulation. Phys. Lett. A88: 265-266 
Layne S P, Mayer-Kress G, Holzfuss J 1986 Problems associated with dimensional analysis of electroencephalogram data. In Dimensions and entropies in chaotic systems (ed.) G MayerKress (New York: Springer) pp 246-256

Liebert W, Schuster H G 1989 Proper choice of time delay for the analysis of chaotic time series. Phys. Lett. A142: 107-111

Lin H B 1984 Chaos (Singapore: World Scientific)

Mayer-Kress G, Layne S P 1987 Dimensionality of the human electroencephalogram. In Perspectives in biological dynamics and theoretical medicine (eds) S H Koslow, A J Madel, M F Shlesinger (New York: NY Academy of Sciences) pp 62-87

Nan X, Jinghua X 1988 The fractal dimension of EEG as a physical measure of conscious human brain activities. Bull. Math. Biol. 50: 559-565

Passamante A, Hediger T, Gollub M 1989 Fractal dimension and local intrinsic dimension. Phys.Rev. A39: 3640-3645

Pike E R 1987 Singular system analysis of time data. In Computational systems - natural and artificial (ed.) H Haken (Berlin: Springer Verlag) pp 86-96

Pool R 1989 Is it healthy to be chaotic. Science 243: 604-607

Pradhan N, Narayana Dutt D 1993 A nonlinear perspective in understanding the neurodynamics of EEG. Comput. Biol. Med. 23: 425-442

Rechtschaffen A, Kales A 1968 A manual of standardized terminology, technique and scoring for sleep stages of human subjects. Public Health Services, US Government Printing Office, Washington, DC

Takens F 1981 Detecting strange attractors in turbulence. In Dynamical systems and turbulence. Lecture notes in mathematics (eds) D A Rand, L S Young (Berlin, Heidelberg, New York: Springer) 898: 366-381

Theiler J 1986 Spurious dimension from correlation algorithms applied to limited time-series data. Phys.Rev. 34: 2427-2432

Thomson J M T, Stewart H B 1987 Nonlinear dynamics and chaos (New York: Wiley)

Watt R C, Hameroff S R 1987 Phase space analysis of human EEG during general anesthesia. In Perspectives in biological dynamics and theoretical medicine (ed.) S H Koslow (New York: NY Academy of Sciences) pp 286-288

Wolf A, Swift J B, Swinney H L, Vastano J A 1985 Determining Lyapunov exponents from a time series. Physica D16: 285-317 